\title{
Gastric Motility in Parkinson's Disease is Altered Depending on the Digestive Phase and Does Not Correlate with Patient-Reported Motor Fluctuations
}

\author{
Laura Ruck $^{\mathrm{a}, 1}$, Marcus M. Unger ${ }^{\mathrm{a}, 1, *}$, Jörg Spiegel ${ }^{\mathrm{a}}$, Jan Bürmann ${ }^{\mathrm{a}}$, Ulrich Dillmann ${ }^{\mathrm{a}}$, \\ Klaus Faßbender ${ }^{\mathrm{a}}$, Wolfgang Reith ${ }^{\mathrm{b}}$, Martin Backens ${ }^{\mathrm{b}}$, Ruben Mühl-Benninghaus ${ }^{\mathrm{b}}$ and \\ Umut Yilmaz \\ ${ }^{a}$ Department of Neurology, Saarland University, Kirrberger Strasse, Homburg, Germany \\ ${ }^{\mathrm{b}}$ Department of Neuroradiology, Saarland University, Kirrberger Strasse, Homburg, Germany
}

Accepted 17 July 2020

\begin{abstract}
.
Background: Altered gastric motility is a frequent non-motor symptom of Parkinson's disease (PD). It has been hypothesized that disturbed gastric motility contributes to motor fluctuations in PD due to an erratic gastro-duodenal transport and an unpredictable absorption of drugs.

Objective: We investigated whether patient-reported fluctuations are associated with parameters of gastric motility visualized by real-time magnetic resonance imaging (MRI) of the stomach.

Methods: We analyzed real-time MRI-scans of the stomach after an overnight fasting period in 16 PD patients and 20 controls. MRI was performed 1) in the fasting state, 2) directly after a test meal, and 3) 4 hours postprandially. Gastric motility indices were calculated and compared between groups.

Results: MRI showed an attenuated gastric motility in PD patients compared to controls. The difference was most obvious in the early postprandial phase. Gastric motility was not associated with patient-reported motor fluctuations. Using an iron-containing capsule we were able to retrace retention of drugs in the stomach.

Conclusion: The results of this study stress the importance of considering the phase of digestion when investigating gastric motility in PD. Despite theoretical considerations, we did not find robust evidence for an association between MRI parameters of gastric motility and patient-reported motor fluctuations. For future studies that aim to investigate gastric motility in PD by MRI, we suggest multiple short-time MRIs to better track the whole gastro-duodenal phase in PD. Such an approach would also allow to retrace the retention of drugs in the stomach as shown by our approach using an iron-containing capsule.
\end{abstract}

Keywords: Parkinson's disease, gastrointestinal motility, stomach, real-time magnetic resonance imaging

\footnotetext{
${ }^{1}$ These authors contributed equally to the manuscript

*Correspondence to: Marcus M. Unger, Department of Neurology, Saarland University, Kirrberger Strasse, 66421
}

Homburg, Germany. Tel.: +49 $6841 \quad 16 \quad 24100$; $\quad$ E-mail: marcus.unger@me.com. 


\section{INTRODUCTION}

Gastrointestinal dysfunction, including altered gastric motility, is a frequent and clinically relevant non-motor symptom of Parkinson's disease (PD) and is likely to be caused by alterations in the enteric nervous system, including accumulation of abnormal alpha-synuclein (reviewed by Fasano et al. [1]). Gastrointestinal dysfunction is already reported in subjects at pre-motor stages of PD (REM sleep behavior disorder) [2] as well as in drug-naïve PD patients [3]. An altered gastric motility is associated with disease severity $[3,4]$ and might be worsened by levodopa $[5,6]$.

With regard to the clinical relevance of an altered gastric motility, it is important to note that delayed and erratic gastric emptying might interfere with gastro-duodenal transport and absorption of antiparkinsonian drugs and thereby might contribute to response fluctuations. Motor fluctuations put a great strain on patients and cause a reduction in their quality of life. Therefore, it is of interest to understand the mechanisms that contribute to motor fluctuations.

Different diagnostic techniques can be used to investigate gastric motility, e.g., scintigraphy, breath tests, electrogastrography, antroduodenal manometry, capsule measurement, ultrasound, and MRI. Different techniques have already been applied and have shown gastrointestinal dysfunction in PD [3-5, 7-9]. Each technique has specific advantages and disadvantages and throws light on one particular aspect of gastric motility. In contrast to the widely applied breath tests (that provide an indirect approximation of gastric emptying and have shown a delayed gastric emptying time in PD; reviewed by Knudsen et al. [10]), real-time MRI directly visualizes the peristaltic waves of the stomach and provides the opportunity to quantitatively assess the magnitude of contractions as well as the velocity of peristaltic waves. In brief, gastric real-time MRI provides a good spatial and temporal resolution of gastric motility. Gastric realtime MRI has been shown to be a reliable marker of motility (compared with the gold standard scintigraphy) [11], has the advantage of being non-invasive, radiation-free and widely available. Moreover, gastric MRI is a versatile approach that allows investigation of different functional aspects (gastric emptying, gastric accommodation, intragastric food distribution, etc.) in one single examination. Gastric real-time MRI has been applied in different conditions $[12,13]$ and has proven feasible also in PD visualizing an impaired motility in PD patients $[8,9]$. So far, one real-time MRI study of gastric motility has been applied as postprandial single-step MRI in PD [9] and one study investigated motility over 120 minutes with 8 sets of scans [8]. The latter study focused on dyspeptic symptoms and did not include healthy controls.

A disturbed gastric motility might contribute to motor fluctuations in PD due to a delayed (and/or erratic) gastro-duodenal transport and absorption of anti-parkinsonian drugs [14].

In this study, we investigated whether MRI parameters of a disturbed gastric motility are associated with the presence and severity of patient reported motor fluctuations in PD. As secondary parameters we investigated gastric motility in PD in different phases of the digestive process and evaluated the suitability of an iron-containing capsule to retrace the gastro-duodenal transport of medication in PD by MRI.

\section{METHODS}

The study was approved by the Ethics Committee of the Medical Association of Saarland (Study No 180/16). Written informed consent was obtained from all subjects prior to enrolment in the study. Subjects were enrolled and investigated between January 2017 and January 2018 at the Department of Neurology and the Department of Diagnostic and Interventional Neuroradiology, Saarland University, Homburg, Germany.

\section{Subjects}

Forty-one subjects were initially enrolled, data of 36 subjects were suitable for analyses (sixteen patients with PD according to the UK Parkinson's Disease Society Brain Bank Clinical Diagnostic Criteria and 20 age-matched controls). Three patients with PD were excluded from the analyses (nonfasted patient $n=1$; quality of MRI-scan insufficient for analysis $n=1$; intake of domperidone $n=1$ ) and 2 control subjects were excluded (positive family history for PD $n=1$; symptoms indicative for early PD $n=1$ ). Legally incompetent subjects and subjects incapable of providing informed consent were excluded by exclusion criteria. Other exclusion criteria were: intake of drugs affecting gastrointestinal motility within 14 days prior to the investigations, presence of endocrine or gastrointestinal disorder which might affect gastric emptying (e.g., diabetic or other polyneuropathy, clinically 
relevant liver or thyroid dysfunction), presence of any contraindication to perform an MRI scan, presence of any chronic or acute disorders of the gastrointestinal tract, history of gastrointestinal surgery (other than appendectomy), pregnancy. For controls the following additional exclusion criteria applied: motor symptoms (e.g., mild isolated tremor, isolated non-bothersome gait disturbances or mild isolated bradykinesia) or symptoms indicative for REM sleep behavior disorder as a non-motor symptoms suspicious of incipient PD, presence of any other neurodegenerative disorder, family history of neurodegenerative disorders.

All subjects underwent a standardized interview concerning past medical history and patient files were cross-checked for conditions/medications that might interfere with the investigations. Presence of constipation was captured by establishing medical history (fewer than three bowel movements a week, or hard, dry and small bowel movements that are painful or difficult to pass, i.e., unsatisfactory defecation). All subjects underwent a medical and neurological examination including collection of the following items/scores by the same investigator (LR) that has been trained in UPDRS scoring: body mass index, Hoehn and Yahr staging, UPDRS scoring (in ON stage), Parkinson Neuropsychometric Dementia Assessment (PANDA), Mini-Mental State Examination (MMSE), Parkinson's Disease Questionnaire (PDQ-39) and Wearing-Off questionnaire (WOQ9). To control for PD-related medication, levodopa equivalent daily doses (LEDD) were calculated based on current recommendations [15]. All PD patients were outpatients at our clinic; PD subtype was defined by the respective treating physicians who were all board-certified neurologists with special expertise in movement disorders.

In addition, PD patients were asked to document motor fluctuations or dyskinesia in a standardized protocol for 24 hours every 30 minutes.

\section{Test meal and gastric real-time MRIs}

All subjects underwent a first MRI scan (interdigestive phase) at $7 \mathrm{AM}$ after a 12-hour overnight fasting period. PD patients underwent the first MRI scan prior to the first intake of their regular antiparkinsonian medication (except for 2 patients who accidently took their medication prior to the first MRI scan). Directly after the first MRI had been performed, PD patients were instructed to take their regular morning medication. All subjects received a standardized semi-solid test meal after the first MRI scan. The test meal consisted of a bread roll with butter and cheese and $250 \mathrm{ml}$ multi-fruit juice (equivalent to approximately 2,916 kilojoule; 72\% carbohydrates, $14.5 \%$ fat and $13.5 \%$ protein). In addition to the test meal, all subjects took a capsule with 283.83 mg ferrous-(II)-glycine sulphate-complex. After the test meal, subjects underwent a second MRI scan (early postprandial phase; median time after the test meal: 24 minutes \pm 15 minutes for PD patients and $30 \pm 11$ minutes for controls). A third MRI scan was performed approximately 4 hours after the test meal (late postprandial phase; median time after the test meal: 274 minutes \pm 23 minutes for PD patients and $261 \pm 29$ minutes for controls). All subjects were instructed to drink maximally $200 \mathrm{ml}$ between scan 2 and 3 and to refrain from eating and physical activity. Medication was taken according to the individual medication plan.

All subjects were examined in the supine position on a 1.5 T MRI scanner (Magnetom Symphony Tim, Siemens Healthcare, Erlangen, Germany). At each of the three points in time, TRUFI (True Fast Imaging with Steady Precession) sequences were obtained in sagittal, axial and coronal plane to localize the position and spatial orientation of the stomach. Next, an individual 20-layer HASTE (Half-Fourier Acquisition Single-Shot Turbo Spin-Echo) sequence was planned and used to identify the layer showing the maximum length expansion of the stomach. This layer was used to obtain a live image TRUFI MRI sequence consisting of 25 sequential breathtriggered recordings. This means that the recordings were made according to the respiratory cycle of the subject and always at the same time of the respiration. This standardization was necessary to avoid respiratory artifacts when analyzing the data.

\section{Analyses of MRI scans}

MRI scans were analyzed with the software OsiriX Lite (Pixmeo SARL, Bernex, Switzerland). Velocity $(\Delta \mathrm{V})$ and amplitude $(\Delta \mathrm{d})$ of individual peristaltic waves in the respective phases were measured. These parameters were then used to calculate the gastric motility index (GMI) for each phase of digestion according to the following formula:

$$
\operatorname{GMI}\left(\mathrm{mm}^{2} / \mathrm{s}\right)=\Delta \mathrm{V} \times \Delta \mathrm{d}
$$

In addition to the calculation of the GMI, the retention of the iron capsule in the stomach was 
evaluated on each MRI scan. All MRI parameters were determined by consensus of two independent investigators (LR and UY). One of the investigators (UY) was a board-certified neuroradiologist and was blinded to all clinical data, including group assignment.

\section{Statistical analyses}

Statistics was carried out using IBM SPSS statistics version 23.0 (SPSS Inc., Chicago, IL, USA). Subjects were assigned to either the PD group or the control group. PD patients were subgrouped in PD patients with motor fluctuations and PD patients without motor fluctuations. Kolmogorow-Smirnow test indicated that there was no normal distribution of our main parameters. Hence, for comparison of continuous variables the non-parametric Mann-Whitney $\mathrm{U}$ test was used. For comparison of binary variables the chi-squared test was used. Bonferroni correction was applied to correct for multiple comparisons. For exploratory analyses of possible associations of demographic and clinical parameters (age, body mass index, medication, motor impairments, etc.) with MRI parameters uncorrected $\mathrm{p}$ values are reported. Correlation was investigated by calculating Spearman's rank correlation coefficient. For correlation of non-motor symptoms related to the gastrointestinal tract with MRI measures, we created a sum score consisting of the following three items: 1) (at least rare) choking when swallowing food; 2) loss of appetite, nausea, vomiting or early upper abdominal fullness; 3) presence of constipation.

\section{RESULTS}

Demographic and clinical data of the enrolled subjects are summarized in Table 1a. Besides a higher body mass index ( $\mathrm{p} 0.04$ ), a higher prevalence of a positive family history for neurodegenerative disorders ( $p$ 0.02) and constipation ( $p$ 0.01) in the PD group, both groups were comparable (Table 1a). Study-related procedures were tolerated well by all investigated subjects.

Gastric real-time MRI showed an attenuated gastric contractility (gastric motility index, GMI) in PD patients compared to age- and sex-matched controls. The difference was most obvious (and statistically significant, $\mathrm{p}$ 0.01) in the early postprandial phase (Fig. 1). PD patients showed a non-significant trend for lower gastric motility indices also in the fasting state and the late postprandial phase (see Supplementary Table 1a).

The velocity of peristaltic waves did not differ between PD patients compared to matched controls. The amplitude of peristaltic waves (as second parameter used to calculate the GMI) showed a statistically significant difference both in the early $(\mathrm{p} 0.015)$ and in the late ( $\mathrm{p} 0.025$ ) postprandial phase, but no difference in the fasting state (see Supplementary Table 1a).

PD patients with motor fluctuations had higher LEDD ( $p$ 0.04), higher total UPDRS (part I, II, III) scores (p 0.02), higher UPDRS part IV scores $(p<0.01)$ and higher scores in the WOQ-9 scores (p 0.03) compared to PD patients without motor fluctuations.

MRI parameters of gastric motility descriptively showed lower GMIs and lower amplitudes in PD patients with reported motor fluctuations compared to PD patients without reported motor fluctuations. Yet, none of the MRI parameters was statistically different between the two PD groups for any of the three investigated points in time (see Supplementary Table 1b).

The GMI showed a significant correlation with the $\mathrm{BMI}$ in the fasting state ( $\mathrm{r}-0.44, \mathrm{p} 0.008)$. An analysis of variance for the two parameters (GMI and BMI) showed no significant result ( $\mathrm{p}$ 0.057). No significant correlation was observed between GMI and BMI in the early $(\mathrm{r}-0.27, \mathrm{p} 0.12)$ or late postprandial phase ( $r-0.09, p$ 0.59). Presence of non-motor symptoms of the gastrointestinal tract correlated with the amplitude ( $\mathrm{r}-0.43, \mathrm{p} 0.01)$ and the GMI $(\mathrm{r}-0.42, \mathrm{p} 0.01)$ of the second MRI. There was no significant correlation between GMI and age, GMI and disease duration, GMI and LEDD or GMI and total UPDRS score, any of the UPDRS subparts respectively, for any of the three investigated points in time. The only significant correlation was observed between disease duration and the velocity of peristaltic waves in third MRI $(\mathrm{r}-0.716$, $\mathrm{p} 0.003)$; this was confirmed in a regression analysis ( $\mathrm{p}$ 0.002). We observed no correlation between disease duration and MRI measures in the fasting state (first MRI) or in the early postprandial phase (second MRI). There was also no correlation between MRI parameters and smoking status or history of appendectomy.

The iron-containing capsule that was coadministered with the test meal (and that is almost identical in shape and in size with capsules containing levodopa) could be easily retraced on the MRI scans as a cruciform artifact (Fig. 2). In the second MRI scan, the capsule was retraced in the 
Table 1a

Demographic and clinical data of analyzed subjects

\begin{tabular}{|c|c|c|c|}
\hline & $\begin{array}{l}\text { PD patients } \\
\quad(n=16)\end{array}$ & $\begin{array}{l}\text { controls } \\
(n=20)\end{array}$ & $p$ value \\
\hline age in years (median [range]) & $62[44-77]$ & $60[41-75]$ & p 0.26 \\
\hline sex (male/female) & $11 / 5$ & $12 / 8$ & \\
\hline $\begin{array}{l}\text { body mass index in } \mathrm{kg} / \mathrm{m}^{2} \text { (median } \\
\text { [range]) }\end{array}$ & $29.2[19.5-35.9]$ & 25 [19.8-31.9] & p 0.04 \\
\hline $\begin{array}{l}\text { disease duration in months (median } \\
\text { [range]) }\end{array}$ & $102.5[23-228]$ & not applicable & \\
\hline PD subtype & $\begin{array}{c}\text { akinetic-rigid: } 9 \\
\text { mixed: } 3 \\
\text { tremor-dominant: } 4\end{array}$ & not applicable & \\
\hline motor fluctuations present & 8 of 16 & not applicable & \\
\hline $\begin{array}{l}\text { levodopa equivalent daily dose in } \mathrm{mg} \\
\text { (median [range]) }\end{array}$ & $550[120-1130]$ & not applicable & \\
\hline $\begin{array}{l}\text { UPDRS score (part I, II, III) (median } \\
\text { [range]) }\end{array}$ & 31.5 [10-91] & $1[0-5]$ & $\mathbf{p}<0.01$ \\
\hline PANDA score (median [range]) & 25 [22-29] & $27[18-30]$ & p 0.15 \\
\hline MMSE score (median [range]) & $30[27-30]$ & $30[27-30]$ & p 0.30 \\
\hline smoker & 1 of 16 & 2 of 20 & $\mathrm{p} 0.65$ \\
\hline history of appendectomy & 4 of 16 & 6 of 20 & p 0.67 \\
\hline history of constipation & 9 of 16 & 3 of 20 & p 0.01 \\
\hline
\end{tabular}

stomach of 33 of the 36 investigated subjects. In the third MRI scan the iron-containing capsule was retraced in the stomach in 6 control subjects and 3 patients with PD (2 PD patients without reported motor fluctuations and 1 PD patients with reported motor fluctuations). An exploratory analysis (using chi-squared test) revealed no significant association between the following factors and the retrieval of the iron-containing capsule on MRI: constipation, smoking, history of appendectomy, reported motor fluctuations.

\section{DISCUSSION}

This study investigated gastric motility by realtime MRI in three different phases of digestion. The difference between PD patients and controls was most obvious (and statistically significant) in the early postprandial phase. When we excluded the data of the two PD patients who accidently took their medication prior to the first MRI scan for an additional analysis (data not shown), all reported significant results still remained significant. On the one hand, this finding confirms and reproduces the results of an initial pilot study in PD. On the other hand, this finding also stresses the need of considering different digestive phases independently when evaluating gastric motility. Cho and colleagues have also shown that timing of MRI after a test meal affects measures of gastric motility in PD [8]. Cho and colleagues focused on dyspeptic symptoms and performed multiple scans up to two hours after a test meal [8], our study provides complementary information for the late postprandial phase (up to four hours after the test meal) and also included controls as reference group.

Gastroduodenal transport also depends on the composition of the respective meal. Solids and liquids as well as macronutrients (e.g., proteins, carbohydrates and fats) affect gastric motility differently. Hence, we composed a standardized test meal consisting of solids and liquids as well as balanced amounts of macronutrients to mimic a standard meal. Admittedly, this standardization with regard to macronutrients might be different from the individual's eating habits and consequently gastric motility might be different for some subjects under real-life conditions. Considering the half-life of dopamine receptor agonists, we cannot completely exclude drug-effects on MRI parameters. On the other hand, all PD patients were on a non-ergoline dopamine receptor agonist therapy (and none of the patients was on an ergoline dopamine receptor agonist). Despite different substances and different daily doses this fact reduces the likelihood of a systematic bias.

The GMI (gastric motility index) is an established overall measure of gastric motility defined by the amplitude and the velocity of peristaltic waves. In order to reveal the exact mechanism that is responsible for our observation, we also analyzed these two factors (i.e., amplitude and velocity of peristaltic waves) independently. In accordance with our pilot study [9], the altered gastric motility was caused by a 

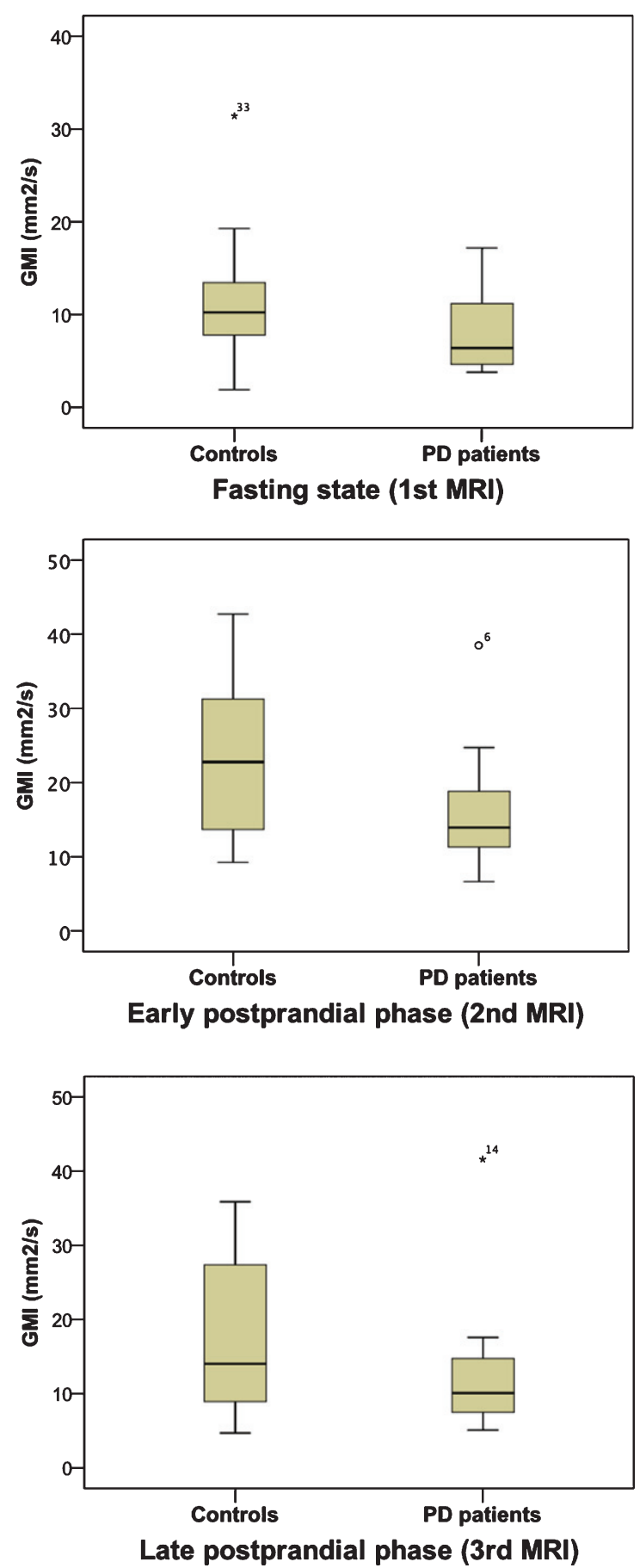

Fig. 1. Gastric motility indices (GMI) for controls and PD patients before (1st MRI) and after (2nd and 3rd MRI) a standardized test meal visualized as boxplots.

decrease in the amplitude of the peristaltic waves and not by an altered velocity of the peristaltic waves. So far, there are only sparse data concerning a differentiation between the velocity and the amplitude of peristaltic waves in patients with gastroparesis. Borovicka and colleagues reported that patients with diabetes mellitus and gastroparesis show increased amplitudes after application of a prokinetic drug [13]. Ajaj and colleagues report a reduced propagation, i.e., velocity of peristaltic waves, in patients with gastroparesis [16] and also an altered propagation of peristaltic waves after administration of motility-modifying drugs in healthy subjects [17]. In accordance with our observation in PD, Janzen et al. showed on a congress poster a decreased amplitude of peristaltic waves by real-time MRI in PD patients after a test meal [18]. Interestingly, a reduced amplitude was also reported for patients with rapid eye movement sleep behavior (a potential pre-motor stage of PD) by this group [18].

Also in the previously cited study by Cho and colleagues [8], the difference in the amplitude of the peristaltic waves was the main contributor for differences in GMI in PD patients (YJL, personal communication, May 2020). Hence, there seems to be a distinct pathophysiological mechanism (altered contractility and not altered propagation) that accounts for the disturbed gastric motility in PD that might be already present in pre-motor stages.

PD patients with self-reported motor fluctuations showed lower mean GMIs compared with PD patients without self-reported motor fluctuations for all three investigated points in time. Yet, the difference was not statistically significant. Hence, in contrast to our assumption we found no robust evidence for a difference between the two PD groups. Indeed, an altered gastroduodenal transport of drugs is not necessarily the sole cause for motor fluctuations in PD, CNS factors might contribute to motor fluctuations as well. In addition, we defined the two subgroups by the overall presence of motor fluctuations. Future studies should also investigate the association of MRI measures with more specific motor complications, e.g., PD patients with delayed-on. The lack of a significant difference observed in this study might also be due to an overall change in gastric motility that was not tracked by the three MRI scans performed in this study. Multiple short-time MRIs might be more suitable to track the whole gastro-duodenal phase in PD. Indeed, peristaltic waves of a single subject are likely to vary even in a defined digestive phase. In this study, we analyzed the peristaltic wave that could be observed best over the longest possible segment in the recorded sequences. Nevertheless, operational matters limited the duration each subject spent in the MRI scanner and therefore also the number of investigated waves. 
Table $1 b$

Demographic and clinical data of PD patients with and without motor fluctuations

\begin{tabular}{|c|c|c|c|}
\hline & $\begin{array}{l}\text { PD patients with } \\
\text { motor fluctuations }(n=8)\end{array}$ & $\begin{array}{l}\text { PD patients without } \\
\text { motor fluctuations }(n=8)\end{array}$ & \\
\hline age in years (median [range]) & $60.5[44-77]$ & $63.5[50-75]$ & $\overline{\mathrm{p} 0.72}$ \\
\hline sex (male/female) & $4 / 4$ & $7 / 1$ & \\
\hline $\begin{array}{l}\text { body mass index in } \mathrm{kg} / \mathrm{m}^{2} \\
\text { (median [range]) }\end{array}$ & $29.7[19.5-35.9]$ & $28.1[23.6-34.1]$ & p 0.72 \\
\hline $\begin{array}{l}\text { disease duration in months } \\
\text { (median [range]) }\end{array}$ & $109.5[50-228]$ & $53[23-172]$ & $\mathrm{P} 0.23$ \\
\hline $\begin{array}{l}\text { levodopa equivalent daily dose in } \\
\mathrm{mg} \text { (median [range]) }\end{array}$ & $785.5[375-1130]$ & 225 [120-900] & p 0.04 \\
\hline $\begin{array}{l}\text { UPDRS score (part I, II, III) } \\
\text { (median [range]) }\end{array}$ & $48.5[20-91]$ & $18.5[10-51]$ & p 0.02 \\
\hline $\begin{array}{l}\text { UPDRS score (part IV) (median } \\
\text { [range]) }\end{array}$ & $4[3-9]$ & $0[0-3]$ & $\mathrm{p}<0.01$ \\
\hline WOQ-9 & $4[1-6]$ & $1[0-5]$ & p 0.03 \\
\hline PANDA score (median [range]) & $26.5[22-29]$ & $23.5[22-28]$ & p 0.79 \\
\hline MMSE score (median [range]) & $29.5[28-30]$ & $30[27-30]$ & p 0.65 \\
\hline
\end{tabular}
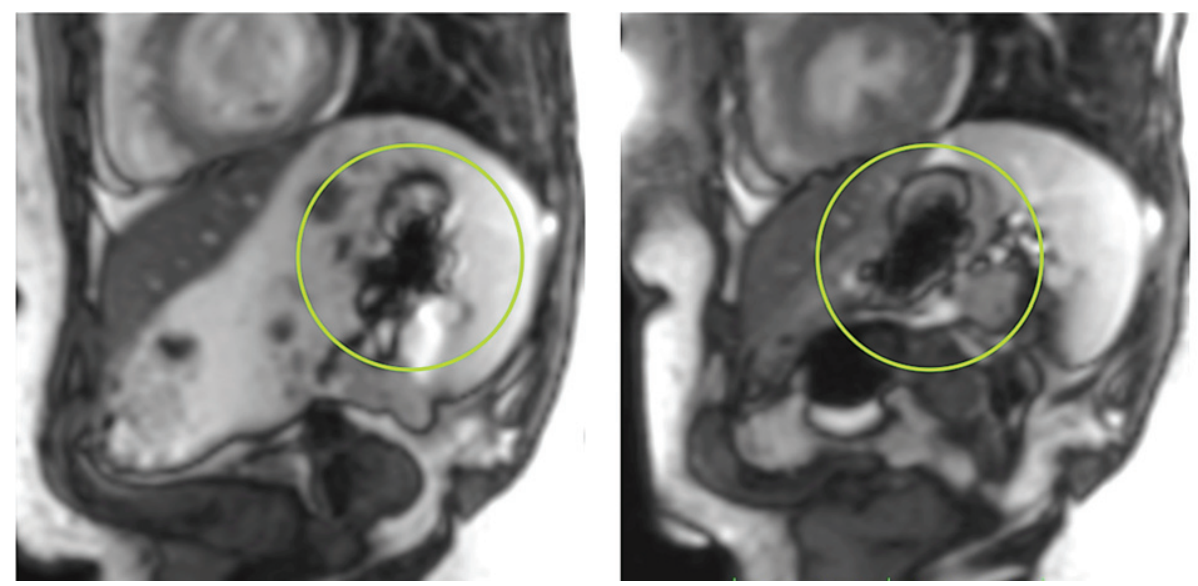

Fig. 2. TRUFI sequences (True Fast Imaging with Steady Precession) in the sagittal plane showing the typical artifact (green circle) of the iron-containing capsule.

Hence, the mean values of each group represent only an approximation of the peristaltic activity in each digestive phase.

A prerequisite for the pharmacological effect of most drugs that are administered to treat PD is the absorption in the small intestine. Hence, gastroparesis and a delayed gastro-duodenal transport can impede the effect of orally administered drugs. A secondary aspect of this study was therefore to determine the feasibility of tracking the retention of drugs in the stomach by MRI. For this purpose we co-administered an enteric-coated capsule with $283 \mathrm{mg}$ ferrous-(II)-glycine sulphate-complex, a capsule that is used for iron supplementation (lowest available dose). In accordance with our assumption, this capsule could be visualized in the stomach in all but 3 investigated subjects by a typical artifact
(Fig. 2). Interestingly, two of the three subjects in whom we were not able to retrace the capsule in the second MRI scan had a longer duration between the ingestion of the test meal and the second MRI when compared to the median of the group (52 minutes, 56 minutes respectively; median PD group: 26 minutes, median control group 19.5 minutes). Hence, the capsule most likely had been already transported to the small intestine in these three subjects. Indeed, traceability of drugs and determination of the gastroduodenal transport is a clinically important aspect in many conditions. For future studies that aim to investigate gastric motility in PD by MRI, we suggest using multi-step MRI of the stomach after ingestion of an iron-containing capsule for this purpose.

The number of subjects that were investigated in this study was limited, partially due to operational 
matters (three different defined time slots for each patient in the morning and at noon to perform an MRI). Hence, the generalizability of our finding is limited due to the limited number of investigated subjects. On the other hand, the data of this study are in accordance with a pilot study in an independent patient cohort. The limited number of subjects in each of the two PD subgroups (with/without motor fluctuations) also limits the representativeness regarding different clinical parameters. Yet, the overall aim of this study was to gain first data on differences in gastric motility between PD patients with and without motor fluctuations without claiming representativeness for each group. This also accounts for the significant difference between PD patients and controls concerning BMI. Despite this significant difference in BMI, BMI was not related with MRI parameters in a regression analysis ( $p$ 0.057). Hence, we conclude that the difference in BMI is unlikely to have systematically biased our results.

In order to avoid respiratory artifacts, we performed breath-triggered recordings (see Methods section). This approach implies that the temporal resolution of the recordings depends also on the individual breathing rate. Acoustical breathing commands might help to standardize breathing rates. Yet, we preferred to not interfere with the individual breathing rate, as it might be difficult for some subjects to comply with acoustical breathing commands and might cause additional movement artifacts. Indeed, movement artifacts are critical when performing imaging of the head/neck or extremities but are a much lesser issue when performing imaging of the torso. We used very fast single shot turbo spin echo sequences that are also used in children, uncooperative patients or fetal imaging.

Retracement of the gastro-duodenal transport of drugs is clinically more relevant than an overall measure of gastric motility in PD. Our study proves the feasibility of easily monitoring gastro-duodenal transport of drugs by MRI without the need for developing specific contrast media. We suggest applying multiple step short-time MRIs with shorter intervals between each MRI and additional sequences to trace the capsule directly after the transport to the duodenum for this purpose.

\section{Acknowledgment}

The publication fee was covered by the non-profit association neuro-g e.V.

\section{Conflict of interest}

The authors have no conflict of interest to report.

\section{SUPPLEMENTARY MATERIAL}

The supplementary material is available in the electronic version of this article: https://dx.doi. org/10.3233/JPD-202144.

\section{REFERENCES}

[1] Fasano A, Visanji NP, Liu LW, Lang AE, Pfeiffer RF (2015) Gastrointestinal dysfunction in Parkinson's disease. Lancet Neurol 14, 625-639.

[2] Knudsen K, Fedorova TD, Hansen AK, Sommerauer M, Haase AM, Svendsen KB, Otto M, Ostergaard K, Krogh K, Borghammer P (2019) Objective intestinal function in patients with idiopathic REM sleep behavior disorder. Parkinsonism Relat Disord 58, 28-34.

[3] Goetze O, Nikodem AB, Wiezcorek J, Banasch M, Przuntek H, Mueller T, Schmidt WE, Woitalla D (2006) Predictors of gastric emptying in Parkinson's disease. Neurogastroenterol Motil 18, 369-375.

[4] Goetze O, Wieczorek J, Mueller T, Przuntek H, Schmidt WE, Woitalla D (2005) Impaired gastric emptying of a solid test meal in patients with Parkinson's disease using 13Csodium octanoate breath test. Neurosci Lett 375, 170-173.

[5] Hardoff R, Sula M, Tamir A, Soil A, Front A, Badarna S, Honigman S, Giladi N (2001) Gastric emptying time and gastric motility in patients with Parkinson's disease. Mov Disord 16, 1041-1047.

[6] Bestetti A, Capozza A, Lacerenza M, Manfredi L, Mancini F (2017) Delayed gastric emptying in advanced Parkinson disease: Correlation with therapeutic doses. Clin Nucl Med 42, 83-87.

[7] Knudsen K, Haase AM, Fedorova TD, Bekker AC, Ostergaard K, Krogh K, Borghammer P (2017) Gastrointestinal transit time in Parkinson's disease using a magnetic tracking system. J Parkinsons Dis 7, 471-479.

[8] Cho J, Lee YJ, Kim YH, Shin CM, Kim JM, Chang W, Park JH (2019) Quantitative MRI evaluation of gastric motility in patients with Parkinson's disease: Correlation of dyspeptic symptoms with volumetry and motility indices. PLoS One 14, $\mathrm{e} 0216396$.

[9] Unger MM, Hattemer K, Moller JC, Schmittinger K, Mankel K, Eggert K, Strauch K, Tebbe JJ, Keil B, Oertel WH, Heverhagen JT, Knake S (2010) Real-time visualization of altered gastric motility by magnetic resonance imaging in patients with Parkinson's disease. Mov Disord 25, 623-628.

[10] Knudsen K, Szwebs M, Hansen AK, Borghammer P (2018) Gastric emptying in Parkinson's disease - A mini-review. Parkinsonism Relat Disord 55, 18-25.

[11] Feinle C, Kunz P, Boesiger P, Fried M, Schwizer W (1999) Scintigraphic validation of a magnetic resonance imaging method to study gastric emptying of a solid meal in humans. Gut 44, 106-111.

[12] Baba S, Sasaki A, Nakajima J, Obuchi T, Koeda K, Wakabayashi G (2009) Assessment of gastric motor function by cine magnetic resonance imaging. J Gastroenterol Hepatol 24, 1401-1406. 
[13] Borovicka J, Lehmann R, Kunz P, Fraser R, Kreiss C, Crelier G, Boesiger P, Spinas GA, Fried M, Schwizer W (1999) Evaluation of gastric emptying and motility in diabetic gastroparesis with magnetic resonance imaging: Effects of cisapride. Am J Gastroenterol 94, 2866-2873.

[14] Djaldetti R, Baron J, Ziv I, Melamed E (1996) Gastric emptying in Parkinson's disease: Patients with and without response fluctuations. Neurology 46, 1051-1054.

[15] Schade S, Mollenhauer B, Trenkwalder C (2020) Levodopa Equivalent dose conversion factors: An updated proposal including opicapone and safinamide. Mov Disord Clin Pract 7, 343-345.

[16] Ajaj W, Goehde SC, Papanikolaou N, Holtmann G, Ruehm SG, Debatin JF, Lauenstein TC (2004) Real time high resolution magnetic resonance imaging for the assessment of gastric motility disorders. Gut 53, 1256-1261.

[17] Ajaj W, Lauenstein T, Papanikolaou N, Holtmann G, Goehde SC, Ruehm SG, Debatin JF (2004) Real-time highresolution MRI for the assessment of gastric motility: Preand postpharmacological stimuli. J Magn Reson Imaging 19, 453-458.

[18] Janzen A, Hasemann T, Paule EK, Bernhard F, Vadasz D, Sittig E, Mayer G, Menzler K, Knake S, Oertel W (2018) Echtzeit-MRT der Magenmotilität bei Patienten mit REM-Schlaf-Verhaltensstörung und de novo ParkinsonKrankheit. Poster neurowoche 2018, P246. 